\title{
Finite Element Analysis Based on Yarn Squeezing Model for the Strength Prediction of Three-dimensional Braided Composites
}

\author{
Long $\mathrm{Hu}^{\mathrm{a}}$, Guoquan Tao, Yibo Wang \\ School of Aeronautic Science and Engineering, Beihang University (BUAA), Beijing 100191, China \\ ahulong0754@qq.com
}

Keywords: 3D braided composites, yarn squeezing effect, continuum damage theory, strength prediction.

\begin{abstract}
A new model considering the yarn squeezing effect in the braided structure of the three-dimensional full five directional (3DF5D) braided composites is established. Repeated unit-cell (RUC) model was used in this model to describe the microstructure of the braided composites. Spatial path and cross-section shape of the yarns in the braided structure are characterized. Damage theory is employed to simulating the damage initiation and evolution in the yarns and resin. A user defined material subroutine (UMAT) based on this theory is developed to be introduced into the finite element analysis model. The damage progression of 3DF5D braided composites RUC under uniaxial tensile loading is simulated. The strength prediction is compared with the experimental data. Good agreement is obtained between the predicted and experiment results.
\end{abstract}

\section{Introduction}

Three dimensional full five directional (3DF5D) braiding composites have higher fiber volume percentage and better mechanical performance for the braided structure than three dimensional four directional (3D4D) or three dimensional five directional (3D5D) braiding composites and have been widely used in many aspects. The 3DF5D concept was first introduced by Liu[1] and then the mesoscopic structures and mechanical properties were investigated by Zhang[2], $\mathrm{Pi}[3]$ and $\mathrm{Xu}[4,5]$.

Investigation on the strength properties of 3D braided composites have been conducted by many researchers. Nonlinear finite element analysis methods were used by Tang and Postle[6] to simulate the deformation of 3D braided composites. Continuum damage theories with different failure criterions and damage evolution laws were introduced into the finite element analyses by Zeng[7] and Fang[8]. Ultimate failure loads and damage behaviors of 3D braided composites under unidirectional tension and compression were predicted and compared with the experimental results.

Local features in the braid structure such as fiber distortion and cross-section shape deformation of yarns due to squeezing effect have been observed by the researchers [9]. Current research works about these features focused on the 3D4D braided composites. However, for the 3DF5D braided composites, the braided structure is more complex and the fiber volume fraction is much higher, resulting in more complicated spatial shape and contact pattern of the yarns. So more and clearer detailed features need to be obtained.

The direct observation data from micro CT scanning for the mesoscopic braid structure of 3DF5D braided composites have been published by $\mathrm{Ya}[10]$. Then the influence of the yarn squeezing effect on the spatial path and cross-section shape of yarns were discussed. Based on these previous works, the present paper established a new mesoscopic simulation model of 3DF5D braided composites, in which the fiber distortion and the change of yarn cross-section shape due to the yarn squeezing effect were considered. Using nonlinear finite element analysis, the ultimate load capacity and damage morphology in the mesoscopic braid structure under uniaxial tension load were investigated. The simulation prediction was compared with the experiment result to validate the accuracy of the simulation model. 


\section{Simulation Model}

According to the braiding procedure, there are two kinds of yarns in the braid structure of 3DF5D braided composites: axial yarn and braiding yarn. It is observed from the micro CT scanning result that the spatial paths of these yarns are generally straight line. The cross-section shapes of braiding yarns barely change in different locations inside the braid structure, as shown in Fig. 1(a). However, a clear changing tendency can be observed from the cross-section shape of the axial yarns in the micro CT scanning images, as shown in Fig. 1. Apparently, this periodic variation characteristic is due to the uneven squeezing effect between the axial yarns and braiding yarns. According to the geometrical characteristics, the cross-section shape of braiding yarn in interior region can be assumed to be a constant hexagonal, as shown in Fig. 1(a). The geometric morphology of the axial yarn can be seen as a square cylinder with periodic uplifts on its surfaces, as shown in Fig. 1(b).

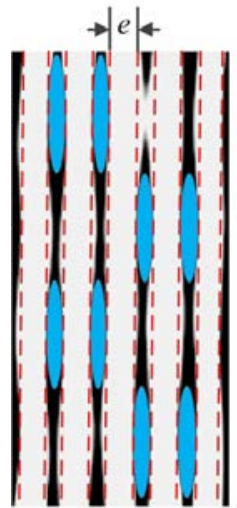

(a)

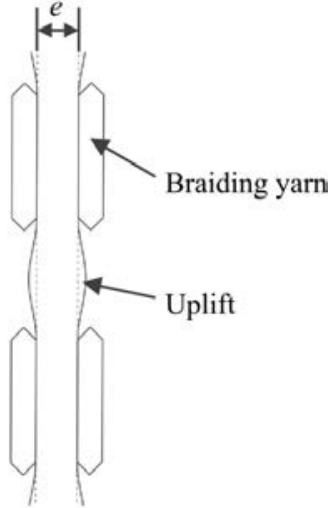

Fig. 1 Geometry morphology of the axial yarn(a) and braiding yarn(b)

Based on the above Geometric models of the yarns, a new RUC models of 3DF5D braided composites with consideration of the yarn squeezing effect was built, as shown in Figure 4. Good agreement on the changing tendency of the axial yarn cross-section shape between the RUC model and the micro-CT scanning images is shown. The squeezing effect on the geometric morphology of the axial yarns is clearly expressed.
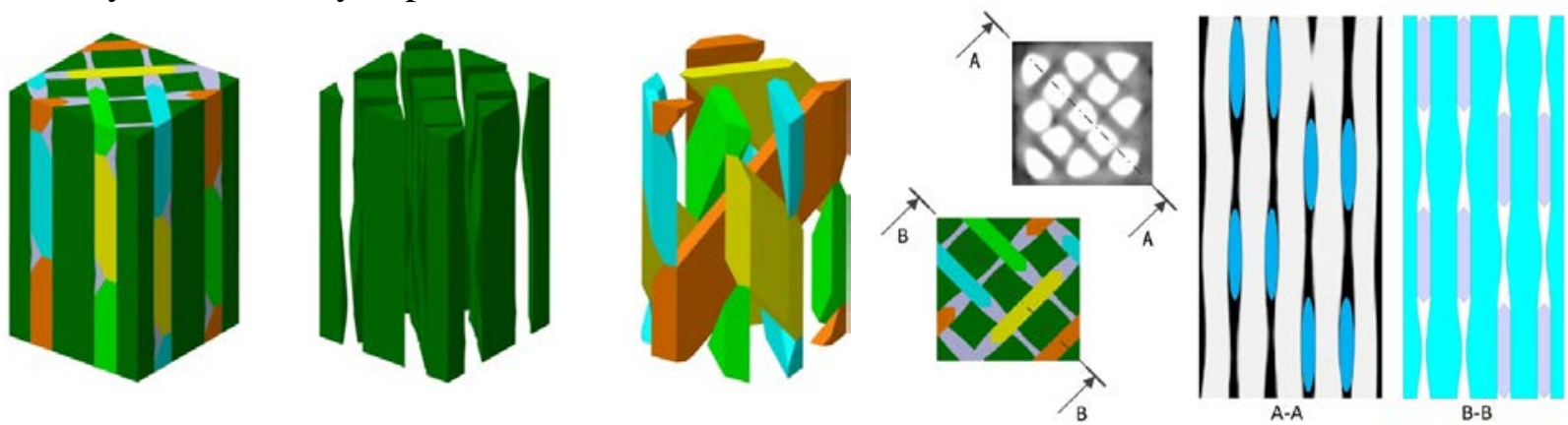

Fig. 2 RUC model of 3DF5D braided composites and comparison with micro CT scanning results

\section{Damage Theory}

The failure criterion of the yarns used in this paper is the 3D Hashin failure criterion, which categorizes the failure mechanisms into two modes: yarn longitudinal failure and yarn transverse failure. Longitudinal tensile failure criterion is expressed as:

$$
f_{\mathrm{Lt}}=\left(\frac{\sigma_{\mathrm{L}}}{X_{\mathrm{L}}^{\mathrm{t}}}\right)^{2}+\alpha\left(\frac{\sigma_{\mathrm{LT}}}{S_{\mathrm{LT}}}\right)^{2}+\alpha\left(\frac{\sigma_{\mathrm{ZT}}}{S_{\mathrm{ZT}}}\right)^{2}=1, \quad \sigma_{\mathrm{L}} \geq 0
$$

Longitudinal compressive failure is expressed as: 


$$
f_{\mathrm{Lc}}=\left(\frac{\sigma_{\mathrm{L}}}{X_{\mathrm{L}}^{\mathrm{c}}}\right)^{2}=1, \quad \sigma_{\mathrm{L}}<0
$$

The tensile and compressive failure are also considered in transverse failure criterion.

Transverse tensile and shear failure:

$$
f_{\mathrm{T}(\mathrm{Z}) \mathrm{t}}=\left(\frac{\sigma_{\mathrm{T}}+\sigma_{\mathrm{Z}}}{X_{\mathrm{T}}^{\mathrm{t}}}\right)^{2}+\frac{\sigma_{\mathrm{TZ}}^{2}-\sigma_{\mathrm{T}} \sigma_{\mathrm{Z}}}{\left(S_{\mathrm{TZ}}\right)^{2}}+\left(\frac{\sigma_{\mathrm{LT}}}{S_{\mathrm{LT}}}\right)^{2}+\left(\frac{\sigma_{\mathrm{ZT}}}{S_{\mathrm{ZT}}}\right)^{2}=1, \quad \sigma_{\mathrm{T}}+\sigma_{\mathrm{Z}} \geq 0
$$

Transverse compressive and shear failure:

$$
f_{\mathrm{T}(\mathrm{Z}) \mathrm{c}}=\frac{1}{X_{\mathrm{T}}^{\mathrm{c}}}\left[\left(\frac{X_{\mathrm{T}}^{\mathrm{c}}}{2 S_{\mathrm{TZ}}}\right)^{2}-1\right]\left(\sigma_{\mathrm{T}}+\sigma_{\mathrm{Z}}\right)+\left(\frac{\sigma_{\mathrm{T}}+\sigma_{\mathrm{Z}}}{2 S_{\mathrm{TZ}}}\right)^{2}+\frac{\left(\sigma_{\mathrm{TZ}}^{2}-\sigma_{\mathrm{T}} \sigma_{\mathrm{Z}}\right)}{\left(S_{\mathrm{TZ}}\right)^{2}}+\left(\frac{\sigma_{\mathrm{LT}}}{S_{\mathrm{LT}}}\right)^{2}+\left(\frac{\sigma_{\mathrm{LZ}}}{S_{\mathrm{LZ}}}\right)^{2}=1, \sigma_{\mathrm{T}}+\sigma_{\mathrm{Z}}<0
$$

In the above equations, $X_{\mathrm{L}}^{\mathrm{t}}, X_{\mathrm{T}}^{\mathrm{t}}$ are tensile strengths in the longitudinal and transverse direction of yarn. $X_{\mathrm{L}}^{\mathrm{c}}, X_{\mathrm{T}}^{\mathrm{c}}$ are compressive strengths in the longitudinal and transverse direction of yarn. $S_{\mathrm{LT}}, S_{\mathrm{LZ}}$ and $S_{Z T}$ are LT, TZ and ZL shear strengths. $\alpha$ is the contribution factor in the failure modes.

\section{Numerical Simulation}

To validate the accuracy of the simulation model and investigate the yarn squeezing effect on the strength property of the 3DF5D braided composites, a finite element model based on RUC is built using finite element software package ABAQUS/Standard, where the load steps, contact property and boundary conditions are set. The damage theory is involved into this model by developing a user defined material subroutine. The mesh scheme of the simulation model is shown in Fig. 3.
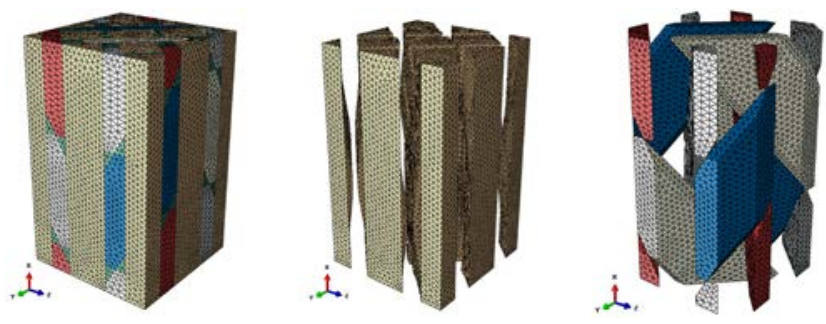

Figure 3 mesh scheme of the simulation model

The failure and progressive damage process of the 3DF5D RUC model under uniaxial tensile load is simulated. The predicted stress-strain curve is shown in Fig. 4 and compared with the experiment result. It is seen from the figure that the material exhibits linear elastic characteristics before the occurrence of damage. When the initial failure occurs, the axial stress decreases rapidly with the increase of strain, and the material quickly loses its bearing capacity and exhibits brittle fracture. The comparison between the numerical prediction and experimental results show that the relative errors are within an acceptable level. Good agreement can be seen between the predicted and experimental results. 


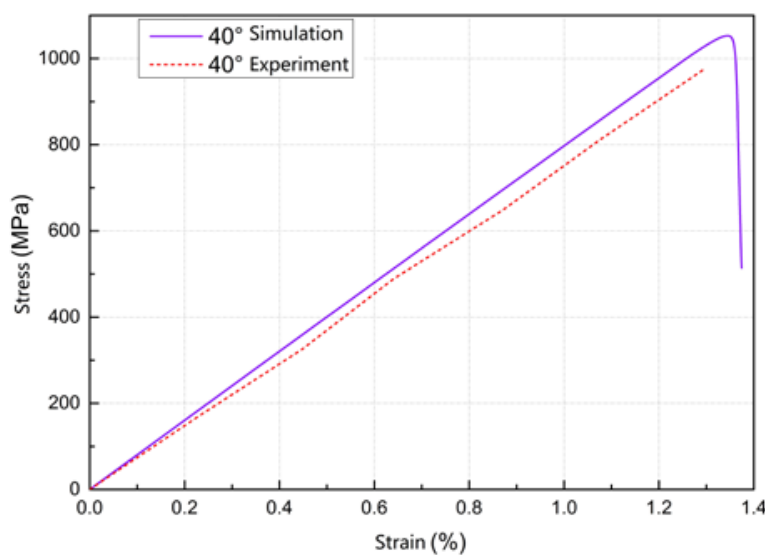

Fig. 4 the predicted and experimental stress-strain curve under uniaxial tensile load

The damage distribution and evolution process of the yarns of the 3DF5D braided composites are shown in Fig.5. It is seen from the figure that after the initial damage occurs, the damage region expands along the cross-section of the axial yarn. When the material reaches the ultimate failure stress, the damage variables of most elements in the damage area increase rapidly to over 0.9 , which means that the elements enter a completely failed state. This in turn causes loss of the longitudinal load carrying capacity of the axial yarn. The damage area of the braided yarn has, however, not been significantly expanded. The damage variables of the elements in the damaged area are also small and most of which are lower than 0.2 .

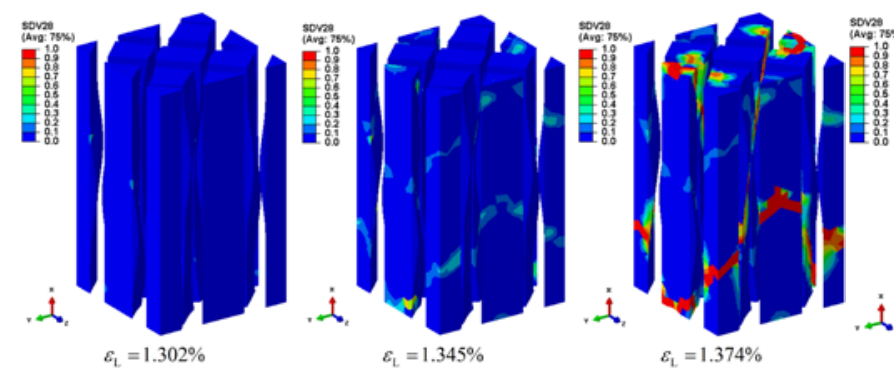

(a)

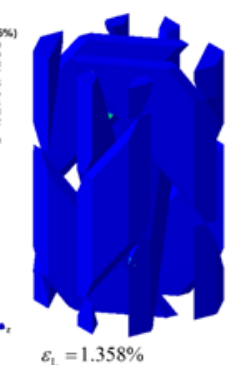

$\varepsilon_{1}=1.358 \%$

Fig. 5 The damage distribution and evolution process of the axial yarn(a) and braiding yarn(b).

\section{Conclusion}

The strength property of the 3DF5D braided composites with consideration of the yarn squeezing effect is explored. A new simulation model is built, in which the fiber distortion and the yarn cross-section deformation due to the squeezing effect are characterized based on micro CT scanning results. Then the load capacity and damage evolvement of the 3DF5D braided composites under uniaxial tensile load are analyzed based on this model. The simulation results are compared with the experimental results provided by former researchers and good agreement is obtained.

\section{References}

[1] Liu, Z.: Concept of three-dimensional all five-directional braided performs. Mater Sci Eng S. 1 (2008) 305-312.

[2] Zhang, F., Liu, Z., Wu, Z., Tao, G.: A new scheme and microstructural model for 3D full 5-directional braided composites. Chinese Journal of Aeronautics 23(1) (2010) 61-67.

[3] Zhang, C., Xu, X., Chen, K.: Application of three unit-cells models on mechanical analysis of 3D five-directional and full five-directional braided composites. Applied Composite Materials 20(5) (2013) 803-825. 
[4] Xu, K., Qian, X: A new analytical model on predicting the elastic properties of 3D full five-directional braided composites based on a multiunit cell model. Composites Part B: Engineering 83 (2015) 242-252.

[5] Xu, K., Qian, X.: Microstructural modeling of three-dimensionally full five-directional braided composites based on a new multiunit cell scheme. Journal of Industrial Textiles 46(2) (2016) 388-416.

[6] Tang Z X, Postle R, Mechanics of three-dimensional braided structures for composite materials-part III: nonlinear finite element deformation analysis, Compos Struct, vol. 55(3),(2002) 307-317.

[7] Zeng T, Wu L, Guo L, A damage model for 3D braided composites with transverse cracking, Compos Struct, vol. 62(2) (2003) 163-170.

[8] Guo-dong F, Jun L, Bao-lai W, Progressive damage and nonlinear analysis of 3D four-directional braided composites under unidirectional tension, Compos Struct, vol. 89(1) (2009) 126-133.

[9] Zhu, Y., Cui, H., Wen, W., Xu, Y., Zhang, H. Microstructure model and stiffness prediction of 3D braided composites considering yarns' cross-section variation. Acta Materiae Compositae Sinica 29(6) (2012) 187-196.

[10] Ya, J., Liu, Z., Wang, Y. Micro-CT Characterization on the Meso-Structure of Three-Dimensional Full Five-Directional Braided Composite. Applied Composite Materials 24(3) (2017) 593-610. 